\title{
Current guidelines for nut consumption are achievable and sustainable: a hazelnut intervention
}

\author{
S. L. Tey ${ }^{1}$, R. Brown ${ }^{1 *}$, A. Chisholm ${ }^{1}$, A. Gray $^{2}$, S. Williams ${ }^{2}$ and C. Delahunty ${ }^{3}$ \\ ${ }^{1}$ Department of Human Nutrition, University of Otago, PO Box 56, Dunedin, New Zealand \\ ${ }^{2}$ Department of Preventive and Social Medicine, University of Otago, PO Box 56, Dunedin, New Zealand \\ ${ }^{3}$ CSIRO, Division of Food and Nutritional Sciences, PO Box 52, North Ryde, NSW 1670, Australia \\ (Received 24 August 2010 - Revised 12 November 2010 - Accepted 16 November 2010 - First published online 28 January 2011)
}

\section{Abstract}

Nuts are known for their hypocholesterolaemic properties; however, to achieve optimal health benefits, nuts must be consumed regularly and in sufficient quantity. It is therefore important to assess the acceptability of regular consumption of nuts. The present study examined the long-term effects of hazelnut consumption in three different forms on 'desire to consume' and 'overall liking'. A total of forty-eight participants took part in this randomised cross-over study with three dietary phases of 4 weeks: $30 \mathrm{~g} / \mathrm{d}$ of whole, sliced and ground hazelnuts. 'Overall liking' was measured in a three-stage design: a pre- and post-exposure tasting session and daily evaluation over the exposure period. 'Desire to consume' hazelnuts was measured during the exposure period only. Ratings were measured on a $150 \mathrm{~mm}$ visual analogue scale. Mean ratings of 'desire to consume' were 92 (SD 35) $\mathrm{mm}$ for ground, 108 (sD 33) mm for sliced and 116 (SD 30) mm for whole hazelnuts. For 'overall liking', the mean ratings were 101 (SD 29) $\mathrm{mm}$ for ground, 110 (SD 32) mm for sliced and 118 (SD 30 ) $\mathrm{mm}$ for whole hazelnuts. Ground hazelnuts had significantly lower ratings than both sliced $(P \leq 0 \cdot 034)$ and whole hazelnuts $(P<0 \cdot 001)$, with no difference in ratings between sliced and whole hazelnuts $(P \geq 0 \cdot 125)$. For each form of nut, ratings of 'overall liking' and 'desire to consume' were stable over the exposure period, indicating that not only did the participants like the nuts, but also they wished to continue eating them. Therefore, the guideline to consume nuts on a regular basis appears to be a sustainable behaviour to reduce $C V D$.

\section{Key words: Compliance: Guidelines: Nuts: Liking: CVD}

CVD, in particular heart attack and stroke, contributes to onethird of all global deaths and one-tenth of the total global burden of disease ${ }^{(1)}$. It is the leading cause of death and disability in many developed countries and is increasing in developing countries ${ }^{(2,3)}$. Diets rich in nuts with favourable fatty acid profiles and other bioactive substances have been shown to reduce cholesterol and thus lower the risk of $\mathrm{CVD}^{(4-7)}$. In New Zealand, as in many other countries, there is a recommendation to consume one serving $(30-42.5 \mathrm{~g})$ of nuts/ $\mathrm{d}^{(8,9)}$. Several epidemiological studies and clinical trials have consistently demonstrated a cardioprotective and a cholesterol-lowering effect with this amount of nuts ${ }^{(10-13)}$. To achieve optimal health benefits, nuts must be consumed regularly and in sufficient quantity. Thus, it is important to determine whether the regular consumption of raw, unsalted nuts is a sustainable long-term behaviour.

In order to measure long-term compliance to a dietary guideline, it is important to measure both 'liking' and 'desire to consume'. 'Liking' is the immediate experience or anticipation of pleasure derived from the orosensory stimulation of eating a food (e.g. 'it tastes nice'), and 'desire to consume' is the intrinsic motivation to engage in eating a food, now or in the near future (e.g. 'I want to consume the food') ${ }^{(14,15)}$. Prolonged exposure to a food may affect these two measures differently. For instance, when consuming a particular food for a prolonged period, liking may remain unchanged; however, the desire to consume the food may be lower. This scenario will then reduce the compliance to regular consumption of the food. For example, Liem \& Zandstra $^{(16)}$ reported that the 'desire to consume' small-shaped snacks decreased significantly, whereas the liking remained unchanged after the daily consumption of the snacks for 3 weeks, which suggests that repeated daily consumption of snacks affects wanting rather than liking in these children.

Numerous studies have been carried out to investigate the effects of repeated exposure to foods on change in liking in adults. These studies have suggested that repeated exposure can induce change in liking, in either a neutral, positive or a negative direction, and several factors may influence this change. Zajonc ${ }^{(17)}$ proposed the theory of 'mere exposure', whereby the liking of a food product is enhanced or reinforced with repeated exposure ${ }^{(18,19)}$. This is especially 
apparent when the initial liking for the food product is low ${ }^{(20-23)}$ and when the food product and stimuli are unfamiliar ${ }^{(24-28)}$. In contrast, the liking of more familiar products or foods with good initial liking often remains constant ${ }^{(29-33)}$ or declines ${ }^{(27,34-38)}$ with repeated consumption. Product boredom or monotony is used to describe the decline in acceptance over days or weeks with repeated exposure caused by loss of interest in specific attributes of the food product ${ }^{(39,40)}$.

Despite the public health recommendation to consume nuts daily ${ }^{(9,41-43)}$, and studies demonstrating the importance of incorporating nuts into the diet for beneficial effects on cardiovascular risk factors ${ }^{(44,45)}$, only one study has investigated the effects of incorporating nuts into the diet on hedonic ratings ${ }^{(29)}$. The hedonic ratings remained unchanged after consuming peanuts for 19 weeks. However, this study only measured the ratings at baseline and at the end of the study ${ }^{(29)}$. Therefore, there is no information on the changes that might have occurred over the duration of the study. In addition, 'desire to consume' was not measured, which means that although liking remains stable, whether individuals still wish to consume the nuts regularly remains unknown.

It was hypothesised that regular consumption of $30 \mathrm{~g}$ of nuts/d may result in boredom and reduce the motivation to comply with the public health guideline. Hence, 'desire to consume' nuts may reduce over time. It was also hypothesised that 'overall liking' for whole hazelnuts may be higher than sliced and ground hazelnuts due to the familiarity of whole nuts. 'Overall liking' for sliced and ground hazelnuts, which are less familiar to the participants, may increase over time due to the 'mere exposure' effect. On the other hand, 'overall liking' for whole hazelnuts, which are more familiar, may decrease over time due to the 'monotony' effect. The primary objective of the present study was to determine whether the current guideline regarding the regular consumption of $30 \mathrm{~g} / \mathrm{d}$ of nuts is an achievable public health goal. Second, does the form of nut influence compliance with the recommendations?

\section{Methods}

\section{Study design}

Most previous studies investigating the effects of repeated exposure to different food items have employed betweensubject designs, and only a few have used within-subject designs $^{(22,23,34,37)}$. The present study was part of a larger nutrition intervention that investigated the cholesterol-lowering effects of different forms of nuts for which a within-subject design was chosen ${ }^{(46)}$. This design allows participants to act as their own controls, permitting a smaller overall sample size, with greater gains in this respect where the correlation between repeated measures is higher. However, it does assume a lack of carry-over effects and places a higher demand on participants. Incorporating a sensory component into a nutrition intervention in this case has allowed us to not only measure health effects of nut consumption, but has also enabled us to investigate whether the public health recommendation to consume nuts regularly is a sustainable behaviour.

The study was conducted over a 4-month period using a randomised cross-over design with three dietary phases: ground, sliced and whole hazelnuts. Participants were randomly allocated to receive one of the three forms of hazelnuts for a period of 4 weeks. Each nut phase was followed by a 2-week washout period. The National Heart Foundation of New Zealand recommends the consumption of $30 \mathrm{~g}$ of nuts, 5 times/week ${ }^{(9)}$. Following this guideline, the participants in the present study were asked to consume $30 \mathrm{~g}$ of hazelnuts each day in place of a high-saturated fat snack or 'treat food'. Participants were asked not to consume additional nuts or nut products during the study. A booklet containing hazelnut recipes and information about the study was given to all participants in order to provide a variety of ways in which nuts can be consumed and, thus, to improve participants' compliance during the 12 -week intervention period.

\section{Participants}

A total of forty-eight participants (twenty males, twenty-eight females) were recruited from the general public in Dunedin, New Zealand. The recruitment process involved the distribution of flyers around the University of Otago campus and in local supermarkets, advertising in the local newspaper and by writing to former research participants who had consented to be contacted about future research projects. Participants were excluded if they had asthma, food allergies, familial hyperlipidaemia, a chronic disease, or were taking medication that was known to affect blood lipid concentrations. Due to personal issues unrelated to the study, two participants (one female and one male) dropped out of the study.

The present study was conducted according to the guidelines laid down in the Declaration of Helsinki, and all procedures involving human subjects were approved by the Human Ethics Committee of the University of Otago, New Zealand. Written informed consent was obtained from all participants. The trial was registered for inclusion in the Australian Clinical Trials Registry and the allocated code ACTRN: ACTRN012607000178448.

\section{Hazelnuts}

Raw, unsalted Ennis hazelnuts were purchased from Uncle Joe's Walnuts (Blenheim, Marlborough, New Zealand). The nuts were shelled and delivered to the researcher as whole nuts a week before the intervention began in order to ensure freshness of the nuts. All the nuts used in the present study were stored at room temperature in darkness before opening. To obtain sliced hazelnuts, the whole hazelnuts were mechanically processed with the use of a food processor (Robot Coupe CL50; Robot Coupe USA, Inc., Jackson, MS, USA). To produce ground hazelnuts, the whole hazelnuts were initially ground using a food processor (Tasin 102; Ta Shing Food Machineries Company Limited, Taichung, Taiwan, ROC), and a Waring blender was then used to 
finely ground the hazelnuts. A mechanical sieving process was carried out to ensure that the hazelnuts were finely ground with a particle size $<0.5 \mathrm{~mm}$.

\section{Compliance}

Participants received a 4-week supply of hazelnuts, which were individually portioned into daily serving size bags $(30 \mathrm{~g} / \mathrm{bag})$, at the beginning of each dietary phase. Compliance was assessed by measuring participants' plasma $\alpha$-tocopherol concentration, by weighing the bags returned at the end of each intervention phase and by completion of the home-use sensory test ballot each day during each experimental period.

\section{Procedure}

'Overall liking' ratings of the three different forms of hazelnuts were measured using a three-stage design: a pre-exposure tasting session; a 12-week home-use exposure ballot; a postexposure tasting session. During the pre-exposure tasting session, participants evaluated their 'overall liking' of three forms of hazelnuts. This was followed by the home-use exposure period where participants rated their 'desire to consume' and 'overall liking' for hazelnuts every day during the dietary intervention. The post-exposure tasting session was carried out immediately after the completion of the intervention period.

Pre-exposure session. Participants attended a preexposure tasting session at the sensory laboratory, Department of Food Science, University of Otago. Participants were given a detailed briefing regarding the nature of the study before testing began. Participants were informed about the nutritional content and health benefits of the nuts and the importance of complying with the instructions to consume the daily allowance of hazelnuts.

The tasting session involved a 15 min product assessment acceptability test. Participants were seated in individual sensory booths and presented with three forms of hazelnuts in duplicate (six products in total). Participants were not informed that they would taste the same sample twice. The hazelnuts for the tasting sessions were stored at room temperature. All samples were randomly coded with three-digit numbers and presented simultaneously in $35 \mathrm{ml}$ portions served in clear plastic cups, with lids to preserve freshness. The order of presentation of all samples was balanced among participants to account for order and carry-over effects $^{(47)}$.

Participants were asked to rinse their mouth with water before starting the session. They were instructed to evaluate the samples one at a time in the order presented. They were asked to take one whole nut or one spoonful of the sample into their mouth and swallow the sample after chewing. After tasting each sample, participants were instructed both verbally and in writing to rate their 'overall liking', on a $150 \mathrm{~mm}$ visual analogue scale anchored with 'dislike extremely' on the left-hand side $(0 \mathrm{~mm})$, 'neither like nor dislike' in the middle $(75 \mathrm{~mm})$ and 'like extremely' on the right-hand side of the scale $(150 \mathrm{~mm})$. Participants were given a glass of filtered water at room temperature and unsalted water crackers in order to cleanse the palate. They were instructed to rinse their mouth with water between samples and wait for $1 \mathrm{~min}$ before tasting the next sample. Participants were asked not to converse during testing.

Home-use exposure period. Participants were randomly assigned to one of six possible sequences of the three treatment diets (ground, sliced and whole hazelnuts). The order of receiving the three treatments differed among participants and was counterbalanced among participants to reduce order effects ${ }^{(47)}$. The participants were instructed to consume the daily allotment of hazelnuts at any time of the day and where they wanted (e.g. at home or at work). They were allowed to incorporate the hazelnuts into their meals. Participants were encouraged to consume the sample on one eating occasion and in the form that they were received (i.e. not cooked). Participants were instructed that the nuts were solely for their own personal consumption and should not be shared with others.

During the exposure period, participants were asked to complete a ballot whenever they consumed the nuts. They were first asked to write down the time of day and location where the nuts were consumed. They were then asked to take one nut or one spoonful of the sample and rate their 'desire to consume' on a $150 \mathrm{~mm}$ visual analogue scale, anchored with 'strong desire not to consume' on the lefthand side $(0 \mathrm{~mm})$ to 'strong desire to consume' on the righthand side of the scale $(150 \mathrm{~mm})$. Next, they were instructed to consume the rest of the sample and rate their 'overall liking' on a $150 \mathrm{~mm}$ line scale anchored with 'dislike extremely' $(0 \mathrm{~mm})$, 'neither like nor dislike' $(75 \mathrm{~mm})$ and 'like extremely' $(150 \mathrm{~mm})$. This scale was the same as that used during the pre-exposure tasting session. 'Desire to consume' and 'overall liking' ratings were measured in order to examine how these two constructs differ among different forms of nuts and change with repeated exposure. Finally, participants were asked to state how the nuts were consumed, whether they were consumed as a snack, as a part of a snack, as a whole meal or as a part of a meal.

Post-exposure session. After 3 months of exposure to three forms of hazelnuts, participants were asked to attend a post-exposure tasting session at the sensory laboratory and repeated the procedure carried out at the pre-exposure tasting session, rating the three samples in duplicate.

\section{Statistical analysis}

The effects of consuming three different forms of hazelnuts on 'desire to consume' and 'overall liking' ratings during repeated exposure were examined. Mean ratings were used as long as these were stable over the exposure periods. Linear mixed models with a random participant effect to account for the underlying correlation between the repeated measures were used to examine the effect of different forms of hazelnuts on mean ratings of 'desire to consume' and 'overall liking'. The models included a term 'period' for assessing the influence of the intervention period (one to three), and this was to 
check whether there were any carry-over effects into subsequent dietary periods. The association between mean ratings of 'desire to consume' and 'overall liking' was assessed using Pearson's correlation coefficient.

Logistic regression with random effects for repeated days within each nut type and repeated nut types within each participant was used to determine whether different forms of nuts were consumed at a different time of the day. Similar models were also used to examine whether the nuts were consumed as a meal or as a snack and whether they were consumed alone or with another food.

The changes in mean 'overall liking' ratings were analysed by comparing 'overall liking' rating at baseline with the 'overall liking' rating at the end of the intervention. Paired $t$ tests were used to compare within-subject differences in 'overall liking' ratings before and after the 3 months hazelnut consumption period. One-way ANOVA models were used to determine if there were statistically significant differences between at least two of the means of the 'overall liking' for the forms of hazelnuts at both pre- and post-exposure tasting sessions. The Scheffe test was used for the adjustment of multiple comparison tests.

All statistical analyses were performed using Stata Intercooled version 9.0 (StataCorp, College Station, TX, USA). All tests were two-sided, with the level of statistical significance set at $5 \%$.

\section{Results}

\section{Participant characteristics}

The participants ranged in age from 25 to 64 years with a mean age of 49.9 (SD 9.4) years. The mean height at baseline was $169 \cdot 2(\mathrm{SD} 8 \cdot 5) \mathrm{cm}$, mean weight was 74.4 (SD 13.1) kg and mean BMI was $25.9(\mathrm{sD} 3.5) \mathrm{kg} / \mathrm{m}^{2}$. In the present study, two participants (4\%) were smokers, four participants $(8 \%)$ had dentures or a partial plate, three $(6 \%)$ had sinus conditions, and one (2\%) was taking medications that might affect the senses, especially taste and smell.

\section{Compliance}

Adherence to all three nut forms was reportedly high: ground hazelnuts, $100 \%$; sliced hazelnuts, $100 \%$; whole hazelnuts, $99.4 \%$, according to both the home-use sensory test ballot and the recount of nut packages. Hazelnuts are a rich source of $\alpha$-tocopherol, which allowed the measurement of plasma $\alpha$-tocopherol concentration to be used as a biochemical marker of hazelnut consumption. Plasma $\alpha$-tocopherol concentration increased significantly after each dietary phase $^{(46)}$.

\section{Relationship between 'desire to consume' and 'overall liking' ratings}

The ratings of 'desire to consume' were significantly positively correlated with 'overall liking' ratings for all forms of hazelnuts $(r \geq 0.872 ; P<0.001$ in all cases; Table 1$)$.
Table 1. Relationship between mean 'desire to consume' and mean 'overall liking' ratings of different forms of hazelnuts during the exposure period

(Mean values and standard deviations)

\begin{tabular}{lrrrrrrrr}
\hline & \multicolumn{2}{c}{$\begin{array}{c}\text { Desire to } \\
\text { consume }(\mathrm{mm})\end{array}$} & & \multicolumn{2}{c}{$\begin{array}{c}\text { Overall liking } \\
(\mathrm{mm})\end{array}$} \\
\cline { 2 - 3 } & Mean & SD & & Mean & SD & Correlation & $P$ \\
\hline Ground & 92.06 & 34.86 & & 100.82 & 29.15 & & 0.872 & $<0.001$ \\
Sliced & 107.67 & 33.11 & & 109.93 & 32.36 & & 0.979 & $<0.001$ \\
Whole & 116.18 & 29.83 & & 117.73 & 30.32 & & 0.974 & $<0.001$ \\
\hline
\end{tabular}

\section{Exposure period - 'desire to consume' ratings}

Participants consumed the hazelnuts within one eating occasion most of the time (99.2\%), and consequently only the 'desire to consume' ratings recorded on the first eating occasion were analysed. The 'desire to consume' each form of hazelnut was found to be stable over the $28 \mathrm{~d}$ exposure period. There was no evidence of a period-by-treatment (nut type) interaction $(P=0.675)$. Therefore, a linear mixed model with just two main effects (nut type, period) and a random participant effect was used. There was a significant difference in the 'desire to consume' the different forms of hazelnuts $(P<0 \cdot 001$; Fig. 1$)$. The 'desire to consume' ground hazelnuts was significantly lower than sliced hazelnuts (mean difference $16.1 \mathrm{~mm} ; 95 \%$ CI $5.5,26 \cdot 6 ; P=0.003$ ) and whole hazelnuts (mean difference $23.5 \mathrm{~mm}$; 95\% CI 13.0 , $34 \cdot 1 ; P<0.001)$ at each dietary phase. There was no statistically significant evidence of differences in 'desire to consume' between sliced and whole hazelnuts (mean difference $7.4 \mathrm{~mm}$; $95 \% \mathrm{CI}-3 \cdot 2,18 \cdot 0 ; P=0 \cdot 170)$.

\section{Exposure period - 'overall liking' ratings}

Ratings of 'overall liking' followed the same pattern as ratings of 'desire to consume' (Fig. 2). Again, the liking for these three forms of hazelnuts was stable over the study period. No evidence of a period-by-treatment (nut type) interaction was found $(P=0 \cdot 426)$. Therefore, a linear mixed model with just two main effects (nut type, period) and a random participant effect was used. In keeping with the 'desire to consume' ratings, the results from the linear mixed model showed that there was a significant difference in the 'overall liking' for different forms of hazelnuts $(P=0 \cdot 001)$. The liking for ground hazelnuts was lower than for sliced and whole hazelnuts by $9.6 \mathrm{~mm}(95 \% \mathrm{CI} 0.7,18.4 ; P=0.034)$ and $16.6 \mathrm{~mm}$ (95\% CI 7.7, 25.4; $P<0.001$ ), respectively. The liking ratings for sliced and whole hazelnuts were not statistically significantly different from each other (mean difference $7.0 \mathrm{~mm}$; $95 \%$ CI $-1.9,15 \cdot 9 ; P=0 \cdot 125)$.

\section{Differences in 'overall liking' ratings between pre- and post-exposure sessions}

Table 2 shows a comparison of the 'overall liking' for the three forms of hazelnuts from the pre- to post-exposure sessions. Paired $t$ tests showed that there was no statistically significant 
Fig. 1. Mean 'desire to consume' different forms of hazelnuts during the $28 \mathrm{~d}$ exposure period. Values are means, compared by using a linear mixed model, adjusted for period and nut type $(P<0.001)$, with standard errors represented by vertical bars. ${ }^{a, b}$ Mean values with unlike letters were significantly different $(P<0.05) . \ldots$, Whole hazelnuts; ........., sliced hazelnuts; $\cdots . . . \cdots . .$, ground hazelnuts.

evidence of differences in 'overall liking' ratings from preexposure to post-exposure sessions for any form of nut ( $P \geq 0 \cdot 216$ in all cases).

However, one-way ANOVA found a statistically significant difference in 'overall liking' between nut types at both preexposure $(P<0.001)$ and post-exposure $(P=0.002)$ sessions. Scheffe's post hoc test showed that the 'overall liking' rating for ground hazelnuts was significantly lower than for sliced hazelnuts (both adjusted $P \leq 0.018$ ) and whole hazelnuts (both adjusted $P \leq 0.006$ ) at both time points. There was no evidence of differences in 'overall liking' between sliced and whole hazelnuts at either time point (both adjusted $P \geq 0 \cdot 717$ ).

\section{Exposure period - ways to consume different forms of} hazelnuts

The ways of consuming the hazelnuts depended on the form that the participants received and were significantly different for the different forms of hazelnuts $(P<0 \cdot 001)$. The estimates for the hazelnuts being consumed alone were 31\% (95\% CI $26,36)$ of ground hazelnuts, $42 \%$ (95\% CI 37, 46) of sliced hazelnuts and 64\% (95\% CI 60, 67) of whole hazelnuts. Thus, $69 \%$ of ground hazelnuts, $58 \%$ of sliced hazelnuts and $36 \%$ of whole hazelnuts were consumed as part of a meal or a snack with other foods. After adjusting for the time effect, the OR for sliced hazelnuts being consumed by themselves compared with ground hazelnuts was 1.18 (95\% CI $1 \cdot 10,1 \cdot 25)$, whole hazelnuts compared with sliced hazelnuts was 1.61 (95\% CI 1.47, 1.75) and whole hazelnuts compared with ground hazelnuts was 1.92 (95\% CI 1.75, 2.08). All represent statistically significant differences $(P<0 \cdot 001)$.

\section{Discussion}

This is the first study to demonstrate that the recommendation to consume nuts on a regular basis is an achievable and

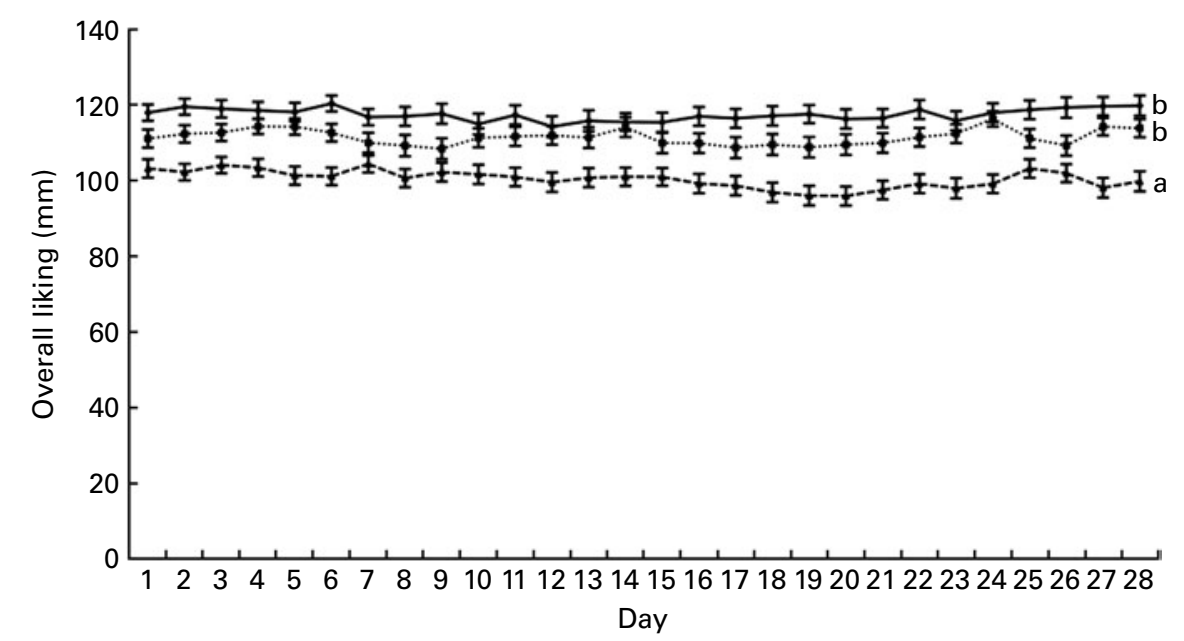

Fig. 2. Mean 'overall liking' ratings of different forms of hazelnuts during the $28 \mathrm{~d}$ exposure period. Values are means, compared by using a linear mixed model, adjusted for period and nut type $(P=0.001)$, with standard errors represented by vertical bars. ${ }^{\mathrm{a}, \mathrm{b}}$ Mean values with unlike letters were statistically significantly different $(P<0.05) . \ldots$, Whole hazelnuts; ........, sliced hazelnuts; ........., ground hazelnuts. 
Table 2. Effect of 3 months of hazelnut consumption on 'overall liking' ratings*

(Mean values with their $95 \%$ confidence intervals, $n$ 46)

\begin{tabular}{|c|c|c|c|c|c|c|c|}
\hline \multirow[b]{2}{*}{ Overall liking } & \multicolumn{2}{|c|}{ Pre-exposure } & \multicolumn{2}{|c|}{ Post-exposure } & \multicolumn{2}{|c|}{ Difference } & \multirow[b]{2}{*}{$P$} \\
\hline & Mean & $95 \% \mathrm{Cl}$ & Mean & $95 \% \mathrm{Cl}$ & Mean & $95 \% \mathrm{Cl}$ & \\
\hline Ground & $92 \cdot 76^{a}$ & $82 \cdot 71,102 \cdot 81$ & $87 \cdot 36^{a}$ & $76.73,97.99$ & $5 \cdot 40$ & $-3 \cdot 27,14 \cdot 08$ & 0.216 \\
\hline Sliced & $109 \cdot 10^{b}$ & $102 \cdot 72,115 \cdot 47$ & $107 \cdot 30^{b}$ & $98.04,116.57$ & $1 \cdot 79$ & $-6 \cdot 32,9 \cdot 90$ & 0.658 \\
\hline Whole & $113 \cdot 67^{b}$ & $107 \cdot 59,119 \cdot 76$ & $110 \cdot 15^{b}$ & $100 \cdot 40,119.91$ & 3.52 & $-5 \cdot 22,12 \cdot 26$ & 0.421 \\
\hline
\end{tabular}

sustainable public health target. This was part of a larger nutrition intervention, which showed that consuming $30 \mathrm{~g}$ of nuts/d for 3 months significantly reduced plasma total cholesterol and increased plasma $\alpha$-tocopherol concentrations ${ }^{(46)}$. In addition to these health benefits, the results of the present study indicate that despite consuming nuts for 3 months, the desire to consume nuts remained unchanged. Regular consumption (i.e. $28 \mathrm{~d}$ each) of three forms of hazelnuts led to a sustained acceptance, suggesting that exposure has no apparent effects on participants' liking. The 'desire to consume' and 'overall liking' ratings for ground hazelnuts were statistically significantly lower than for both sliced and whole hazelnuts, whereas these measurements of the latter two did not differ statistically significantly. The absence of clear changes in 'desire to consume' and 'overall liking' in the present study could be largely attributable to three possible factors.

First, the stable 'desire to consume' and 'overall liking' ratings across the study period may be explained by the attributes of nuts as a food product. It is well documented that repeated exposure to different food products does not affect consumers' acceptance in the same way ${ }^{(39)}$. For instance, repeated consumption did not change the liking of moderately liked staple foods ${ }^{(34-36,40)}$, whereas savoury foods such as plain biscuits ${ }^{(37)}$, chicken soup ${ }^{(27,37)}$, vegetables ${ }^{(36)}$ and meat dishes ${ }^{(36,38)}$ showed a decrease in liking. It seems that nuts could be a food that is resistant to monotony as a product category in general. This is in line with the findings by Alper \& Mattes $^{(29)}$, where consuming peanuts for 19 weeks did not reduce the hedonic ratings (mean rating seven out of nine) from the pre-treatment to the post-treatment session. However, this study did not measure the acceptability ratings during the intervention period. Nevertheless, this is the only study that has reported the acceptability score for nuts after repeated exposure.

Second, earlier work suggests that foods with high initial pleasantness ratings do not develop a lowered acceptance ${ }^{(40,48)}$. The 'overall liking' of all forms of hazelnuts was generally high (above the neutral point, i.e. $75 \mathrm{~mm}$ on the rating scale) at both tasting sessions and during the exposure period. Thus, repeated exposure to all forms of hazelnuts with initially high acceptance ratings did not change participants' 'desire to consume' and 'overall liking' throughout the course of the study. These results are supportive of the findings of previous research in this area, where Hetherington et $a l^{(35)}$ reported that acceptability ratings of a salty high-fat snack food, french fries, with high initial pleasantness ratings remained stable over fifteen exposure periods. However, this finding is not consistent for all foods. Results of three studies have shown that ratings of pleasantness decreased with exposure to a moderately liked sweet high-fat snack food, chocolate, which was not typically consumed regularly on a daily basis ${ }^{(34,35,37)}$. The contradictory results could be due to the differences in taste qualities and macronutrient content, where savoury, high-fat snacks such as unsalted nuts and french fries are more resistant to monotony, while sweet, high-fat snacks such as chocolate are less resistant to monotony $^{(29)}$.

Finally, the choices with regard to how and when a food may be consumed play a major role in determining participants' acceptance. Kramer et al. ${ }^{(49)}$ reported that repeated consumption of the same food with or without choice might yield different results in consumers' acceptance. Studies have shown that the liking ratings for food products were higher in a choice situation than in a no-choice situation ${ }^{(20,21)}$. Results of an in-home exposure test reported that the desire to eat and liking for meat sauce remained unchanged for those participants who were allowed to choose among three flavours of the meat sauce, compared with those who had to consume the meat sauce that was pre-assigned by the researchers ${ }^{(38)}$. Other studies have also shown that lack of choice seems to be the most evident contributor to the boredom effect, and this may have affected the acceptability of the food products $(31,33,35,50)$

In the present study, participants were allowed to consume the hazelnuts at anytime of the day and in any manner that they chose, which meant the participants could consume the hazelnuts in the way they perceived as appropriate. In addition, participants were given a recipe booklet, where suggestions to incorporate different forms of hazelnuts into dishes or daily meals were provided. It is possible that the choices given facilitated the maintenance of acceptance over time. Using logistic regression, there was evidence of differences in the odds of consuming each nut type during the morning (before 11.00 hours, $P<0.001$ with ground $>$ sliced $>$ whole), around noon (11.00 until before 14.00 hours, $P<0.001$ with whole $>$ sliced $>$ ground) and later in the day (14.00 hours and later, $P=0.001$ with whole $>$ ground and whole $>$ sliced). In addition, there was evidence of a difference in consumption as a meal rather than as a snack $(P<0 \cdot 001$, with ground $>$ sliced $>$ whole) and alone rather than with another food 
( $P<0.001$ with whole $>$ sliced $>$ ground). This finding could be built into public health messages, to provide a variety of ways to include nuts into snacks and meals, thus increasing the long-term acceptability of nuts.

To our knowledge, this is the first study to determine the long-term effects ( $28 \mathrm{~d}$ each) of a food product on changes in ratings of 'desire to consume' and 'overall liking' on the visual analogue scale during the exposure period. Both ratings showed similar trends. The number of exposures used to investigate the effect of exposure to a food product on acceptance during repeated consumption has ranged from 3 to $22 \mathrm{~d}$ in previous studies $(18,20-23,25,27,28,30,31,33-38)$. On the other hand, for studies measuring the acceptance of a food product before and after exposure only, the number of exposures ranged from 2 to $7 \mathrm{~d}^{(19,24,26)}$, with the exception of one study that has measured the acceptance after consuming peanuts for 19 weeks $^{(29)}$. It is highly unlikely that nuts require a greater number of exposures to pick up changes in 'desire' and 'overall liking' over time. Thus, a longer time period with more exposure would have been unlikely to change the observation for the present study.

In the present study, the ratings of 'desire to consume' and 'overall liking' for all forms of hazelnuts were found to follow a very similar pattern and were strongly correlated with each other $(r>0 \cdot 80)$. This indicates that not only did participants like the nuts, but also they wished to continue to eat the nuts, despite consuming each form of nuts daily for 4 weeks. The present results agree with previous findings in adults, where high correlations were found between liking and desire-to-eat meat sauce $\left(\begin{array}{rl}r & 0.69\end{array}\right)^{(38)}$, chocolate, bread and butter $(r>0.75)^{(35)}$, and chicken soup $\left(\begin{array}{rl}r & 0.67\end{array}\right)^{(27)}$. Cardello et $a l .^{(51)}$ found a very strong association between the ratings of the expected liking/disliking of foods in different situations and the ratings of their appropriateness for these situations ( $r$ 0.98). As discussed earlier, hazelnuts were probably consumed at a time and in a context that was perceived as most appropriate by the participants, which may have lessened a monotony effect and resulted in high ratings on 'desire to consume' and 'overall liking'. However, direct measurements of participants' perceived boredom or tiredness, and appropriateness to consume hazelnuts each day are required to understand the dynamics of liking and wanting hazelnuts.

The present study investigated not only whether the participants initially liked hazelnuts, but also more importantly whether the participants continued to like nuts after repeated exposure. The findings are strengthened by the fact that the intervention took place in a more naturalistic real-world (home-use) setting rather than in an artificial laboratory setting. A further strength lies in the length of the intervention. This is the first trial that has included a quantitative longterm follow-up and measured the changes in 'desire' and 'liking' of a food product during the exposure period. This has not been done in any previous studies involving nuts. It is clear from the results that a 12 -week trial is a sufficient interval to assess the long-term acceptability of a food product. The findings indicate that participants' liking for hazelnuts as a healthy dietary behaviour is sustainable over time.
One unique aspect of this research is the study design. Most previous studies measuring acceptance and desire to consume have employed a parallel between-group design because of possible carry-over effects. The present study, because it was part of a larger nutritional intervention where participants acted as their own controls, was a multiple cross-over study. We found no evidence of a period-by-treatment interaction, suggesting no carry-over effects in the present study. This indicates that sensory components can be added to nutritional intervention studies where a cross-over design may be preferred due to a smaller overall sample size and high correlations between baseline and follow-up measurements.

However, a limitation of the present study was that it used a convenience sample who were provided with free hazelnuts for 3 months. It is possible that we attracted people who like nuts and may have been more health conscious and motivated than the general population. People with low initial acceptance for nuts may have a different response to acceptance after long-term repeated exposure. Furthermore, the participants in the present study were predominantly New Zealand European adults. It is not possible therefore to state whether the 'desire to consume' and 'liking' would have been similar in children or a more ethnically diverse sample who may have different levels of exposure or experience with nuts. In order to minimise sampling bias, a more representative and diverse subject group with fewer frequent nut eaters could be recruited to permit more definitive conclusions of the effect of long-term nut consumption on acceptance.

\section{Conclusion}

The acceptance for hazelnuts, a nutritionally beneficial highunsaturated fat savoury snack, with reasonably high initial liking was not influenced by long-term consumption of this product. This suggests that hazelnuts are well tolerated on a daily basis when participants are given a choice to consume them in any manner that they wish. It would appear from a public health perspective that the recommendation to consume $30 \mathrm{~g}$ of nuts/d is achievable and acceptable. Whole nuts and sliced nuts were more acceptable than ground nuts, therefore recommendations should favour these forms. However, there are subgroups of the population such as elderly people or those with dentures or a partial plate who may find the nuts with larger particle sizes difficult to consume. Given that the acceptance for all forms of hazelnuts was high, it is advisable to recommend the inclusion of any form of hazelnuts, such as ground, sliced or whole hazelnuts, based on individual's personal preference. This will provide more choices for the consumers, thus enhancing compliance with the recommendation to consume nuts daily as part of a heart-healthy diet.

\section{Acknowledgements}

The funding for the present study was provided by the National Heart Foundation of New Zealand. We would like to thank the participants for their commitment and enthusiasm 
in participating in the present study. None of the authors had any personal or financial conflict of interest. The authors' responsibilities were as follows: S. L. T. designed the study, collected, entered and analysed the data, disseminated the findings, and prepared the manuscript; R. B., A. C. and C. D. were involved in the study design, supervision of data collection, data analysis and preparation of the manuscript; A. G., S. W. were responsible for the assistance with study design, statistical analysis and editing of the manuscript.

\section{References}

1. Yusuf SFD, Reddy SMD, Ounpuu SP, et al. (2001) Global burden of cardiovascular diseases. Part I: general considerations, the epidemiologic transition, risk factors, and impact of urbanization. Circulation 104, 2746-2753.

2. Gazlano TA (2007) Reducing the growing burden of cardiovascular disease in the developing world. Health Aff 26, 13-24.

3. Thom T, Haase N, Rosamond W, et al. (2006) Heart disease and stroke statistics - 2006 update: a report from the American Heart Association Statistics Committee and Stroke Statistics Subcommittee. Circulation 113, E85-E151.

4. Bolling BW, McKay DL \& Blumberg JB (2010) The phytochemical composition and antioxidant actions of tree nuts. Asia Pac J Clin Nutr 19, 117-123.

5. King JC, Blumberg J, Ingwersen L, et al. (2008) Tree nuts and peanuts as components of a healthy diet. J Nutr $\mathbf{1 3 8}$, S1736-S1740.

6. Sathe SK, Monaghan EK, Kshirsagar HH, et al. (2009) Chemical composition of edible nut seeds and its implications in human health. In Tree Nuts: Composition, Phytochemicals, and Health Effects, pp. 12-36 [C Alasalvar and F Shahidi, editors]. Boca Raton, FL: Taylor \& Francis Group.

7. Segura R, Javierre C, Lizarraga MA, et al. (2006) Other relevant components of nuts: phytosterols, folate and minerals. Br J Nutr 96, Suppl. 2, S36-S44.

8. American Institute for Cancer Research \& World Cancer Research Fund (1997) Food, Nutrition and the Prevention of Cancer: A Global Perspective. Menasha, WI: BANTA Book Group.

9. New Zealand Guidelines Group (2003) Evidence-based Best Practice Guideline: The Assessment and Management of Cardiovascular Risk. Wellington: New Zealand Guidelines Group.

10. Griel AE \& Kris-Etherton PM (2006) Tree nuts and the lipid profile: a review of clinical studies. Br J Nutr 96, Suppl. 2, S68-S78.

11. Kelly JH \& Sabate J (2006) Nuts and coronary heart disease: an epidemiological perspective. Br J Nutr 96, Suppl. 2, S61-S67.

12. Kris-Etherton PM, Hu FB, Ros E, et al. (2008) The role of tree nuts and peanuts in the prevention of coronary heart disease: multiple potential mechanisms. J Nutr $\mathbf{1 3 8}$, S1746-S1751.

13. Sabaté J \& Wien M (2010) Nuts, blood lipids and cardiovascular disease. Asia Pac J Clin Nutr 19, 131-136.

14. Mela DJ (2006) Eating for pleasure or just wanting to eat? Reconsidering sensory hedonic responses as a driver of obesity. Appetite 47, 10-17.

15. Zandstra L (2007) Like it or buy it 7th Pangborn Sensory Science Symposium, Minneapolis, MN, USA.

16. Liem DG \& Zandstra LH (2009) Children's liking and wanting of snack products: influence of shape and flavour. Int $J$ Behav Nutr Phys Act 6, 38-47.
17. Zajonc RB (1968) Attitudinal effects of mere exposure. J Pers Soc Psychol 9, 1-27.

18. Luckow T, Sheehan V, Fitzgerald G, et al. (2006) Exposure, health information and flavour-masking strategies for improving the sensory quality of probiotic juice. Appetite 47, 315-323.

19. Stein LJ, Nagai H, Nakagawa M, et al. (2003) Effects of repeated exposure and health-related information on hedonic evaluation and acceptance of a bitter beverage. Appetite 40, 119-129.

20. Chung S-J \& Vickers Z (2007) Influence of sweetness on the sensory-specific satiety and long-term acceptability of tea. Food Qual Prefer 18, 256-264.

21. Chung S-J \& Vickers Z (2007) Long-term acceptability and choice of teas differing in sweetness. Food Qual Prefer 18, 963-974.

22. Essed NH, van Staveren WA, Kok FJ, et al. (2006) The effect of repeated exposure to fruit drinks on intake, pleasantness and boredom in young and elderly adults. Physiol Behav 89, 335-341.

23. Zandstra EH, de Graaf C, Mela DJ, et al. (2000) Short- and long-term effects of changes in pleasantness on food intake. Appetite 34, 253-260.

24. Bingham A, Hurling R \& Stocks J (2005) Acquisition of liking for spinach products. Food Qual Prefer 16, 461-469.

25. Russell K \& Delahunty C (2004) The effect of viscosity and volume on pleasantness and satiating power of rice milk. Food Qual Prefer 15, 743-750.

26. Tuorila H, Meiselman HL, Bell R, et al. (1994) Role of sensory and cognitive information in the enhancement of certainty and linking for novel and familiar foods. Appetite 23, $231-246$.

27. Zandstra EH, Weegels MF, van Spronsen AA, et al. (2004) Scoring or boring? Predicting boredom through repeated in-home consumption. Food Qual Prefer 15, 549-557.

28. Sulmont-Rossé C, Chabanet C, Issanchou S, et al. (2008) Impact of the arousal potential of uncommon drinks on the repeated exposure effect. Food Qual Prefer 19, 412-420.

29. Alper CM \& Mattes RD (2002) Effects of chronic peanut consumption on energy balance and hedonics. Int J Obes Relat Metab Disord 26, 1129-1137.

30. Lévy CM \& Köster EP (1999) The relevance of initial hedonic judgements in the prediction of subtle food choices. Food Qual Prefer 10, 185-200.

31. Stubenitsky K, Aaron JI, Catt SL, et al. (1999) Effect of information and extended use on the acceptance of reduced-fat products. Food Qual Prefer 10, 367-376.

32. Vickers Z \& Holton E (1998) A comparison of taste test ratings, repeated consumption and postconsumption ratings of different strengths of iced tea. J Sens Stud 13, 199-212.

33. Le HT, Joosten M, van der Bijl J, et al. (2007) The effect of NaFeEDTA on sensory perception and long term acceptance of instant noodles by Vietnamese school children. Food Qual Prefer 18, 619-626.

34. Hetherington MM, Bell A \& Rolls BJ (2000) Effects of repeat consumption on pleasantness, preference and intake. $\mathrm{Br}$ Food J 102, 507-521.

35. Hetherington MM, Pirie LM \& Nabb S (2002) Stimulus satiation: effects of repeated exposure to foods on pleasantness and intake. Appetite 38, 19-28.

36. Meiselman HL, de Graaf C \& Lesher LL (2000) The effects of variety and monotony on food acceptance and intake at a midday meal. Physiol Behav 70, 119-125.

37. Weijzen PLG, Zandstra EH, Alfieri C, et al. (2008) Effects of complexity and intensity on sensory specific satiety and 
food acceptance after repeated consumption. Food Qual Prefer 19, 349-359.

38. Zandstra EH, de Graaf C \& van Trijp HCM (2000) Effects of variety and repeated in-home consumption on product acceptance. Appetite 35, 113-119.

39. Moskowitz HR (2000) Engineering out food boredom: a product development approach that combines home use tests and time-preference analysis. Food Qual Prefer 11, 445-456.

40. Siegel PS \& Pilgrim FJ (1958) The effect of monotony on the acceptance of food. Am J Psychol 71, 756-759.

41. Dreher ML, Maher CV \& Kearney P (1996) The traditional and emerging role of nuts in healthful diets. Nutr Rev $\mathbf{5 4}$, 241-245.

42. Haddad EH, Sabate J \& Whitten CG (1999) Vegetarian food guide pyramid: a conceptual framework. Am J Clin Nutr 70, S615-S619.

43. Health Canada (2007) Canada's Food Guide to Healthy Eating. Ottawa: Ministry of Health Canada.

44. Mukuddem-Petersen J, Oosthuizen W \& Jerling JC (2005) A systematic review of the effects of nuts on blood lipid profiles in humans. J Nutr 135, 2082-2089.
45. Sabate J, Oda K \& Ros E (2010) Nut consumption and blood lipid levels: a pooled analysis of 25 intervention trials. Arch Intern Med 170, 821-827.

46. Tey SL, Brown R, Chisholm A, et al. (2010) Effects of different forms of hazelnuts on blood lipids and $\alpha$-tocopherol concentrations in mildly hypercholesterolemic individuals. Eur J Clin Nutr (Epublication ahead of print version 29 September 2010).

47. MacFie HJ, Bratchell N, Greenhoff K, et al. (1989) Designs to balance the effect of presentation and first order carryover effects in hall test. J Sens Stud 4, 129-148.

48. Schutz HG \& Pilgrim FJ (1958) A field study of food monotony. Psychol Rep 4, 559-565.

49. Kramer FM, Lesher LL \& Meiselman HL (2001) Monotony and choice: repeated serving of the same item to soldiers under field conditions. Appetite 36, 239-240.

50. King SC, Meiselman HL, Hottenstein AW, et al. (2007) The effects of contextual variables on food acceptability: a confirmatory study. Food Qual Prefer 18, 58-65.

51. Cardello AV, Schutz H, Snow C, et al. (2000) Predictors of food acceptance, consumption and satisfaction in specific eating situations. Food Qual Prefer 11, 201-216. 